\title{
THE DIMENSION OF THE BOUNDARY OF THE LÉVY DRAGON
}

\author{
P. DUVALL \\ Department of Mathematical Sciences \\ University of North Carolina at Greensboro \\ Greensboro, NC 27412, USA \\ and \\ J. KEESLING \\ Department of Mathematics \\ University of Florida \\ P.O Box 118105,358 Little Hall \\ Gainesville, FL 32611-8105, USA
}

(Received April 7, 1997)

\begin{abstract}
In this paper we describe the computations done by the authors in determining the dimension of the boundary of the Lévy Dragon. A general theory was developed for calculating the dimension of a self-similar tile and the theory was applied to this particular set. The computations were challenging It seemed that a matrix which was $2^{15} \times 2^{15}$ would have to be analyzed. It was possible to reduce the analysis to a $752 \times 752$ matrix. At last it was seen that if $\lambda$ was the largest eigenvalue of a certain $734 \times 734$ matrix, then $\operatorname{dim}_{H}(K)=\frac{\ln (\lambda)}{\ln (\sqrt{(2))}}$. Perron-Frobenius theory played an important role in analyzing this matrix.
\end{abstract}

KEY WORDS AND PHRASES: Hausdorff dimension, iterated function systems, attractors, fractal geometry

1991 AMS SUBJECT CLASSIFICATION CODES: Primary 28A20, 54E40, 57Nxx.

\section{INTRODUCTION}

Self-similar sets have become an important area of research. They are not only interesting geometric figures, but they have also proved to be useful in image compression and storage [1], [2], and [3]. It is possible that those self-similar sets that are tiles might play an important role in image compression in the future. So, these sets deserve to be studied in some detail. The work summarized here is an attempt to extend what is known about these sets.

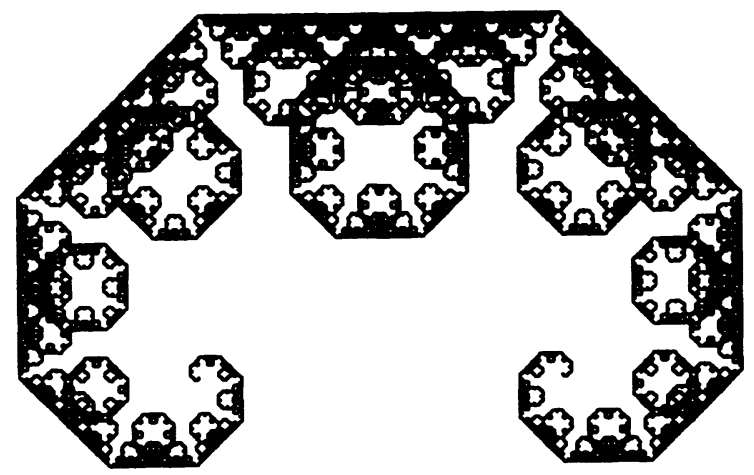

Figure 1. The Lévy Dragon 
In [4], P Lévy studied the complex curve which has come to be known as the Lévy Dragon $\mathrm{He}$ showed that the Dragon is self-similar, and argued that the plane can be tiled by copies of it Lévy's paper is a tour de force on this subject It is amazing that he was able to determine so many properties of this and other curves in [4] without the use of modern computers

Although Levy found many properties of the Levy Dragon, he did not compute the Hausdorff dimension of its boundary. In [5, page 236] G Edgar asks whether the dimension of the boundary of the Dragon is greater than one In a previous paper [6] we answered that question in the affirmative and in fact determined a precise value for this dimension. In the process of doing this we developed a general theory for computing the Hausdorff dimension of the boundary of self-similar fractal tiles

The Lévy Dragon is the attractor for the $\operatorname{IFS} F=\left\{f_{1}, f_{2}\right\}$, where $f_{1}$ and $f_{2}$ are similitudes of Euclidean space $\mathbf{R}^{2}$ given by

$$
\begin{aligned}
& f_{1}(x, y)=\left(\frac{x-y}{2}, \frac{x+y}{2}\right), \\
& f_{2}(x, y)=\left(\frac{x+y+1}{2}, \frac{y-x+1}{2}\right) .
\end{aligned}
$$

Much that we discovered about the Dragon holds in a broader setting So, it is appropriate to have fairly general notational conventions. The Hausdorf dimension of a set $E$ is denoted by $\operatorname{dim}_{H} E$, and the lower and upper box-counting dimensions of $E$ are denoted by $\underline{\operatorname{dim}}_{B} E$ and $\overline{\operatorname{dim}}_{B} E$. Definitions of these dimension functions can be found in [7], [8] and [9].

Let $\Omega$ denote the collection of sequences $I=\left\{i_{1}, i_{2}, \cdots\right\}$ with $i_{k} \in\{1,2, \cdots, n\} . \Omega_{k}$ will denote the set of sequences of length $k$ with entries from $\{1,2, \cdots, n\}$. $\Omega$ has the natural metric given by

$$
d(I, J)= \begin{cases}1, & \text { if } i_{1} \neq j_{1} \\ c_{\imath_{1}} c_{\imath_{2}} \cdots c_{l_{m}}, & \text { if } i_{k}=j_{k} \text { for } k \leq m \text { and } i_{m+1} \neq j_{m+1} .\end{cases}
$$

For $I \in \Omega$, we let $I_{k}$ denote the finite sequence $\left\{i_{1}, i_{2}, \cdots, i_{k}\right\}$, and will often use the shorthand $f_{I_{k}}$ for the composition $f_{i_{1}} f_{2_{2}} \cdots f_{i_{k}}$ and $c_{I_{k}}$ for the product $c_{\imath_{1}} c_{\imath_{2}} \cdots c_{i_{k}}$ We will also need the maps $\sigma: \Omega \rightarrow \Omega$ and $g: \Omega \rightarrow K$ given by $\sigma\left(i_{1}, i_{2}, \cdots\right)=\left\{i_{2}, i_{3}, \cdots\right\}$ and $g(I)=\lim _{k \rightarrow \infty} f_{i_{1}} f_{i_{2}} \cdots f_{z_{k}}(x)$ It is not difficult to see that $g(I)$ is independent of the choice of $x \in X$. The map $\sigma$ is the well-known shift map on the sequence space

\section{SUB-SELF-SIMILAR SETS}

In [7], Falconer gave an (abstract) way to determine the dimension of sub-self-similar sets Given a set $F=\left\{f_{1}, f_{2}, \cdots, f_{n}\right\}$ of contracting similitudes on $\mathbf{R}^{l}$, the closed set $E$ is said to be sub-self-similar for $F$ if $E \subset \bigcup_{\imath=1}^{n} f_{\imath}(E)$. It follows that $E \subset K$, where $K$ is the attractor for $F$. Note in particular that the topological boundary $\partial K$ of $K$ is s.s.s., since the interior int $K$ of $K$ is mapped into itself by each of the open mappings $f_{\imath}$. The first crucial observation about s.s.s. sets is

PROPOSITION 2.1. Let $E$ be a closed set. Then $E$ is s.s.s. for $F$ if and only if $E=g(A)$ for some compact set $A \subset \Omega$ such that $\sigma(A) \subset A$. Furthermore, if $E$ is s.s.s., such a set $A$ is given by $A=\left\{I \in \Omega \mid g\left(\sigma^{k}(I)\right) \in E\right.$ for all $\left.k \geq 0\right\}$.

The set $A$ in Proposition 2.1 is fundamental in the calculation of $\operatorname{dim}_{H} E$. For $k>0$, let $A_{k}$ be the set of finite sequences obtained by truncating elements of $A$ after $k$ terms. For $s \geq 0$ definite

$$
\tau(s)=\lim _{k \rightarrow \infty}\left(\sum_{I_{k} \in A_{k}} c_{I_{k}}^{s}\right)^{1 / k} .
$$

Recall that $F$ satisfies the open set condition [10] if there is a bounded nonempty open set $U$ such that 


$$
\begin{gathered}
\bigcup_{i=1}^{n} f_{\imath}(U) \subset U, \quad \text { and } \\
f_{\imath}(U) \cap f_{j}(U)=\emptyset, \text { if } i \neq j .
\end{gathered}
$$

THEOREM 2.2. Let $E$ be a closed set which is s.s.s. with respect to the family $\left\{f_{1}, f_{2}, \cdots, f_{n}\right\}$ of contracting similitudes which satisfies the open set condition. Let $s$ be the number satisfying $\tau(s)=1$

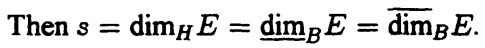

\section{BOUNDARIES OF ATTRACTORS}

One obvious difficulty in applying Theorem 2.2 is that one has to sum over finite sequences determined by an infinite condition It was important to show that membership in $A_{n}$ can be determined from finite data

PROPOSITION 3.1. $A_{k}=\left\{I \in \Omega_{k} \mid f_{I_{k}}(K) \cap \partial K \neq \emptyset\right\}$

Once we had Proposition 3.1 we had a strategy for computing $\operatorname{dim}_{H} \partial K$. Suppose for convenience that the $c_{2}$ all have the same value $c$, and suppose that we can determine $\alpha=\lim _{k \rightarrow \infty}\left|A_{k}\right|^{1 / k}$, where $|S|$ denotes the cardinality of the set $S$. Then

$$
\tau(s)=\lim _{k \rightarrow \infty}\left(\sum_{I_{k} \in A_{k}} c_{I_{k}}^{s}\right)^{1 / k}=\lim _{k \rightarrow \infty}\left(\left|A_{k}\right| c^{k s}\right)^{1 / k}=\alpha c^{s} .
$$

Thus $\operatorname{dim}_{H} \partial K$ is the solution to $\alpha c^{s}=1$, or

$$
\operatorname{dim}_{H} \partial K=-\frac{\ln (\alpha)}{\ln (c)}
$$

\section{THE LÉVY DRAGON}

We now revert to letting $K$ denote the Lévy Dragon with its $I F S\left\{f_{1}, f_{2}\right\}$ Lévy [4] showed, among other things, that $K$ tiles the plane in the sense that the plane can be written as the union of congruent copies of $K$ that meet only in their boundaries.

Let $S$ be the unit square with vertices $\{(0,0),(0,1),(1,1),(1,0)\}$ and let $T_{0} \subset S$ be the triangle with vertices $\{(0,0),(1 / 2,1 / 2),(1,0)\}$. Lévy viewed $K \operatorname{as}_{k \rightarrow \infty} \lim ^{k}\left(T_{0}\right)$.

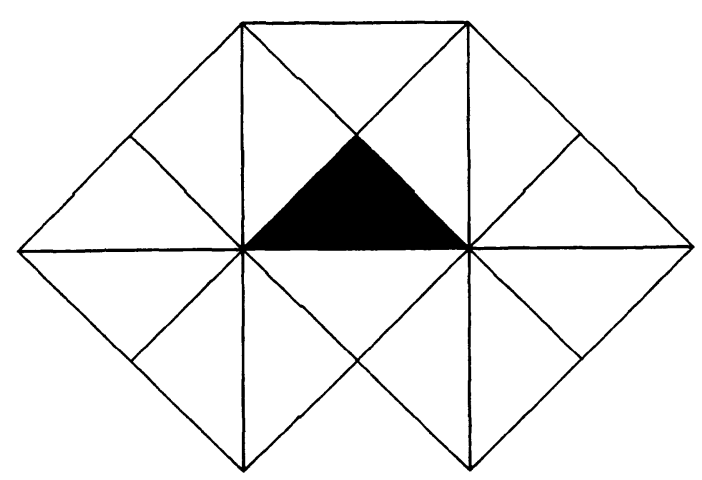

Figure 2. $T_{0}$ and its Neighborhood 
$S$ is the union of four triangles in $S$ with hypotenuse an edge of $S$ and third vertex at $(1 / 2,1 / 2)$ We denote the triangulation of $\mathbf{R}^{2}$ consisting of these triangles and their integral translates by $\mathcal{T}_{0} \quad T_{0}$ and its neighborhood in $\mathcal{T}_{0}$ are shown in Figure 2 If $T$ is a right triangle in the plane, we refer to the vertex opposite the hypotenuse as the top vertex of $T$, and the other vertices as left and right so that we encounter the vertices in the order left, top, and right as we traverse the boundary in clockwise order The left (right) edge of $T$ is the edge determined by the left (right) and top vertices of $T$ The triangulation $\mathcal{T}_{0}$ has a subdivision $\mathcal{T}_{1}$ obtained by subdividing each triangle $T$ into the two triangles determined by the left (right) edge of $T$ and the midpoint of the hypotenuse of $T$ Continuing in the obvious way gives a sequence $\mathcal{T}_{0} \succ \mathcal{T}_{1} \succ \cdots \succ \mathcal{T}_{k} \succ \cdots$ of subdivisions of $R^{2}$ into right isoceles triangles. Each triangle in $\mathcal{T}_{k}$ has diameter $\left(\frac{1}{\sqrt{2}}\right)^{k}$ For each $T \in \mathcal{T}_{k}$, let $T^{L}$ be the triangle in $\mathcal{T}_{k+1}$ whose hypotenuse is the left edge of $T$ and which has a vertex outside of $T$. Define $T^{R}$ similarly. One can easily see that $F\left(T_{0}\right)=T_{0}^{L} \bigcup T_{0}^{R}$. In fact, the following proposition is immediate

PROPOSITION 4.1. For each $k>0, F^{k}\left(T_{0}\right)$ is a union of $2^{k}$ triangles in $\mathcal{T}_{k} . F^{k+1}\left(T_{0}\right)$ is obtained from $F^{k}\left(T_{0}\right)$ by replacing each $T$ in $F^{k}\left(T_{0}\right)$ by $T^{L}$ and $T^{R}$

We used the formula at the end of Section 3 to compute the Hausdorff dimension of $\partial K$ The difficulty that we encountered was that $K$ and $\partial K$ are very complicated objects, and we can only see finite approximations to them, so it was not clear how to compute $A_{k}$ What we were able to work with ' was

$$
B_{k}=\left\{I_{k} \in \Omega_{k} \mid f_{2_{k}}\left(T_{0}\right) \cap \partial F^{k}\left(T_{0}\right) \neq \emptyset\right\}
$$

Then we were able to determine the relationship between $A_{k}$ and $B_{k}$ to complete the calculation

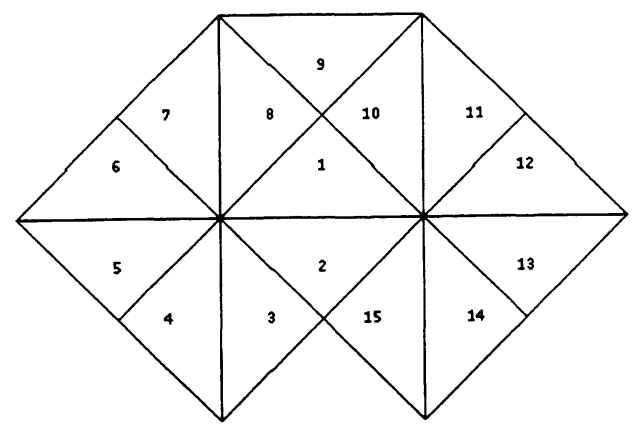

Figure 3 The Neighborhood Ordering

One of the difficulties in dealing with $K$ is that it is not easy to locate points in the interior of $K$ by looking at $F^{k}\left(T_{0}\right)$. One needs to find objects of positive area which persist from iteration to the next, but as soon as a triangle $T$ appears at one level, its interior is discarded at the next level and is replaced by two triangles exterior to $T$. It is not until the iterations have become sufficiently intertwined to create covered triangles that the discarded mass is filled back in by adjacent triangles Through computer experimentation we discovered that covered triangles do not exist until the 14th iteration, and only 8 of the $2^{14}$ triangles that comprise $F^{14}\left(T_{0}\right)$ are covered! However, if a triangle $T \in \mathcal{T}_{k}$ is covered, then $T$ is the union of two triangles in $\mathcal{T}_{k+1}$ which are also covered. It follows that int $T \subset F^{k+m}\left(T_{0}\right)$ for all $m>0$, so that int $T \subset$ int $K$. 


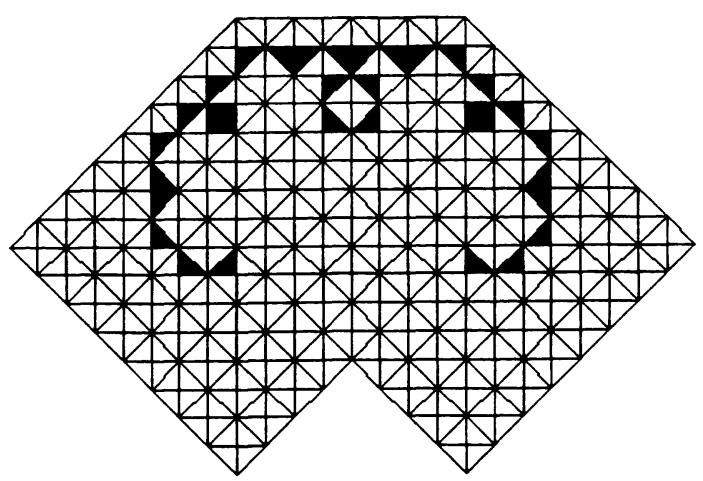

Figure 4. $N_{5}$ and $F^{5} T_{0}$

PROPOSITION 4.2. $B_{k} \subset A_{k}$

It follows that $\left|B_{k}\right| \leq\left|A_{k}\right|$ We also used the fact that at each stage $F^{k}(I)$ is a tiling of $\mathbf{R}^{2}$ So, in using $B_{k}$ two ways, we were able to determine lower and upper bounds for $\operatorname{dim}_{H} \partial K$ which were the same number thus giving us a complete determination of this dimension Let $\beta=\lim _{k \rightarrow \infty}\left|B_{k}\right|^{1 / k}$

\section{THEOREM 4.3.}

$$
\operatorname{dim}_{H} \partial K=\frac{\ln (\beta)}{\ln (\sqrt{2})}
$$

\section{COMPUTATIONAL ISSUES}

In the light of Theorem 4.3, our goal was to understand the asymptotic behavior of the number $\left|B_{k}\right|$ of triangles in $F^{k}\left(T_{0}\right)$ which are not covered. To this end, we needed to study the neighborhood structures introduced in Section 4. In order to keep track of the number of neighborhood types at each stage in $F^{k}\left(T_{0}\right)$ we needed a matrix which was $2^{15} \times 2^{15}$ However, by experimenting with this matrix, we found that we could reduce it to a smaller $734 \times 734$ matrix with nonnegative integer entries which was irreducible Perron-Frobenius theory was used to determine that there was a unique largest eigenvalue $\lambda$ to this matrix. This eigenvalue determined the cardinality of $\left|B_{k}\right|$ to be $\lambda^{k}$ asymptotically This allowed us to compute the dimension of the boundary of the Lévy Dragon We were able to determine precise bounds for $\lambda$ using integer arithmetic and also determine as estimate for $\lambda$ to high precision using the power method.

By Section 4,

$$
\operatorname{dim}_{H} \partial K=\frac{\ln (\lambda)}{\ln (\sqrt{2})} .
$$

Floating point computations using the power method give the estimate $\lambda \approx 1.934007183$ The rigorous estimate that we obtained using integer arithmetic was $1.824190<\lambda<1.974189$. This last estimate proved that $\lambda$ was greater than $\sqrt{2}$, and that the Hausdorff dimension of $\partial K$ is greater than 1 Thus, the boundary of the Dragon has Hausdorff dimension greater than 1, and Edgar's question was answered in the affirmative. 
ACKNOWLEDGEMENT. This research was done while the first author was a Visiting Professor at the University of Florida He gratefully acknowledges UF's hospitality, as well as research support from the University of North Carolina, Greensboro

\section{REFERENCES}

[1] BARNSLEY, M, Fractals Everywhere (2nd ed ), Academic Press, 1993

[2] BARNSLEY, M and HURD, L., Fractal Image Compression, A K. Peters, 1993

[3] FISHER, Y. (ed.), Fractal Image Compression: Theory and Application to Digital Images, Springer-Verlag, 1995

[4] LÉVY, P , Les courbes planes ou gauches et les surfaces composée de parties semblades au tout, Journal de l'École Polytechnique (1938), 227-247, 249-291

[5] LÉVY, P, Plane or space curves and surfaces consisting of parts similar to the whole, Classics on Fractals (G Edgar, ed ), Addison-Wesley, pp 181-239.

[6] DUVALL, $P$ and KEESLING, J The Hausdorff dimension of the boundary of the Lévy Dragon, submitted.

[7] FALCONER, K J, Sub-self-similar sets, Trans. Amer. Math. Soc. 347, 8 (1995), 3121-3129

[8] FALCONER, K J., Fractal Geometry Mathematical Foundations and Applications, John Wiley and Sons, 1990

[9] EDGAR, G A., Measure, Topology, and Fractal Geometry, Springer-Verlag, 1990

[10] HUTCHINSON, J E, Fractals and self similarity, Indiana Univ. Math. J. 30 (1981), 713-747 


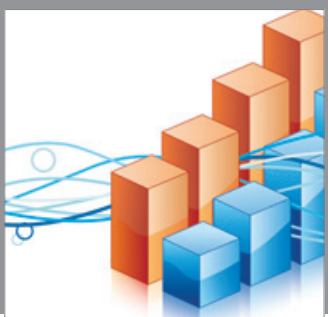

Advances in

Operations Research

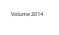

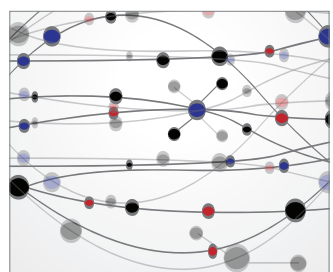

\section{The Scientific} World Journal
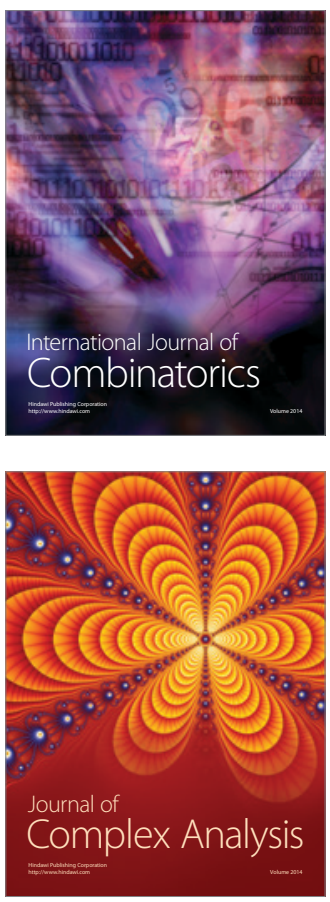

International Journal of

Mathematics and

Mathematical

Sciences
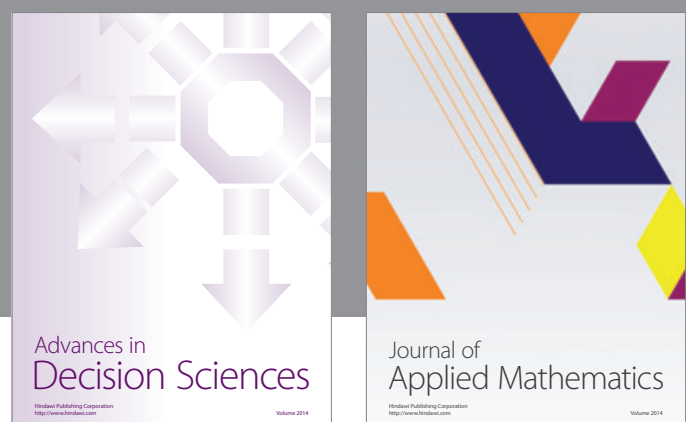

Journal of

Applied Mathematics
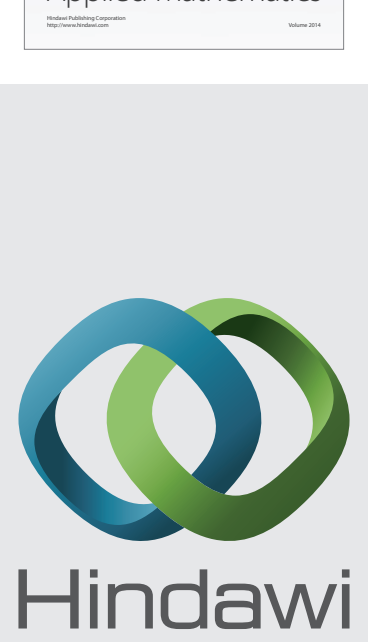

Submit your manuscripts at http://www.hindawi.com
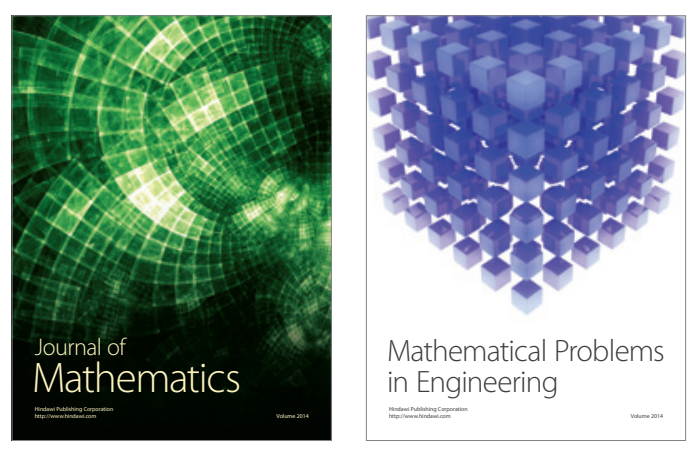

Mathematical Problems in Engineering
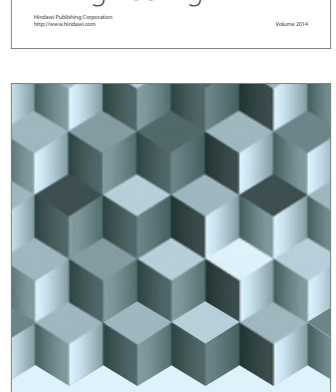

Journal of

Function Spaces
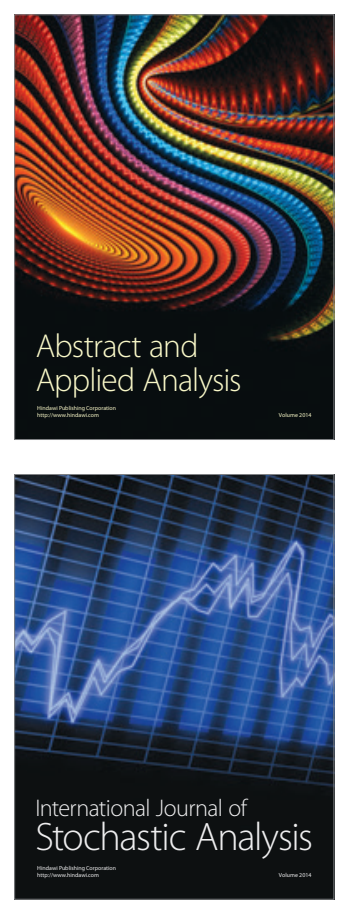

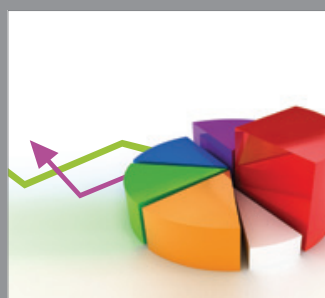

ournal of

Probability and Statistics

Promensencen
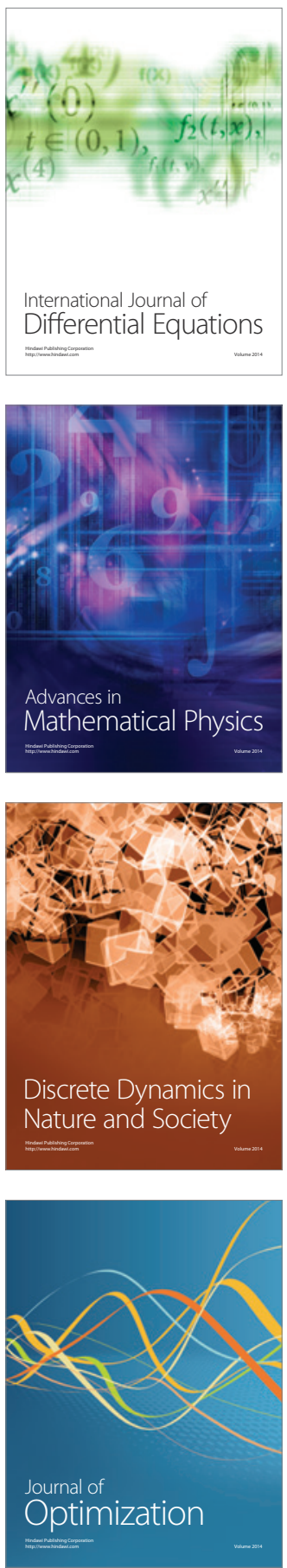